\title{
INNOVATIONEN AN WISSENSCHAFTLICHEN BIBLIOTHEKEN IN ÖSTERREICH: STATUS UND PERSPEKTIVEN AKTUELLER KO- OPERATIONSPROJEKTE
}

\author{
von Bruno Bauer
}

\section{Inhalt}

\section{Einleitung}

2. Innovationsschub durch Kooperationen bei der Transformation von gedruckten (\& elektronischen) Medien zu E-Only

2.1. Transformation der Kataloge

2.2. Transformation der Bibliografien

2.3. Transformation der Zeitschriften

2.4. Transformation von Monografien

3. Desiderate im österreichischen Bibliothekswesen

4. Aktuelle Innovationen durch Kooperationen wissenschaftlicher Bibliotheken in Österreich

4.1. Innovation Suchmaschinentechnologie

4.2. Innovation Bibliothekssystem

4.3. Innovation Kooperation E-Medien Österreich

4.4. Innovation E-Infrastructures Austria

4.5. Innovation Open Access Network Austria

5. Innovationen als Zukunftschance für Bibliotheks- und Informationsinfrastruktur in Österreich

Zusammenfassung: "Innovationen in der Medienwelt" - das Motto der ODOK 2014 findet seine Entsprechung auch in Kooperationsprojekten der wissenschaftlichen Bibliotheken in Österreich. Große Innovationsmöglichkeiten brachte die bereits weitgehend und in Kooperation vollzogene Umstellung von gedruckten auf elektronische Medien mit sich. In jüngster Zeit konnten einige neue zukunftsweisende Kooperationsprojekte gestartet werden, die dazu beitragen werden, die Bibliotheks- und Informationsinfrastruktur in Österreich für Wissenschaft, Forschung und Lehre zu optimieren.

Schlüsselwörter: Österreich; Wissenschaftliche Bibliothek; Kooperation; Status Quo; Perspektive; Eröffnungsvortrag; ODOK 2014 


\section{INNOVATIONS AT ACADEMIC LIBRARIES IN AUSTRIA: STATUS AND PROSPECTS OF CURRENT COOPERATION-PROJECTS}

Abstract: "linnovations in a world of media" - the motto of the 2014 ODOK-conference has its counterparts also within cooperation-projects of Austrian academic libraries. The changeover from printed to electronic media, adjusted largely and in cooperation, has already brought a great deal of possible innovation. Recently several promising cooperation-projects have been started which will contribute to optimize Austrian library and information structures for science, research and teaching.

Keywords: Austria; academic library; cooperation; status quo; prospects; inaugural address; ODOK 2014

\section{Einleitung}

Das Generalthema der ODOK 2014 in Zell am See lautete „Innovationen in der Medienwelt" und lud dazu ein, über innovative Produkte und Dienstleistungen zu berichten, mit denen Bibliothekarinnen und Bibliothekare sowie Informationsspezialistinnen und Informationsspezialisten gemeinsam mit kommerziellen Anbietern den zahlreichen aktuellen Herausforderungen begegnen. In den Mittelpunkt des Call for Papers wurden insbesondere Innovationen in der Erwerbung, in der Erschließung, in der Dokumentation \& Information, in der Medienverwaltung, in der Aus- und Weiterbildung sowie neue Ansätze in der Evaluierung gestellt. ${ }^{1}$

Bemerkenswert ist die Tatsache, dass der Begriff „Innovation“ in den facheinschlägigen Nachschlagewerken des Bibliotheks- und Informationswesens nicht behandelt wird, was zur Annahme verleiteten kann, dass Innovation in diesem Bereich entweder nicht vorkommt oder so selbstverständlich ist, dass der Begriff nicht eigenständig behandelt wird. So etwa ist im „Lexikon für Bibliotheks- und Informationswissenschaft“ nur der Begriff „Innovationsproblem“ aufgenommen, nicht aber „Innovation“ an sich. ${ }^{2}$

Wenn man den Betriff „Innovation“ thematisiert, ist es hilfreich abzuklären, was der Begriff „Innovation“ bedeutet. Innovation, wörtlich „Neuerung“ oder „Erneuerung“, leitet sich vom lateinischen Wort „innovare“ (erneuern) ab. Während „Innovation" umgangssprachlich im Sinn von neuen Ideen und Erfindungen verwendet wird, resultieren, wenn man von Innovation im engeren Sinn spricht, aus Ideen erst dann Innovationen, wenn diese zur Umsetzung neuer Produkte, Dienstleistungen oder Verfahren und zu deren tatsächlicher erfolgreicher Anwendung und Marktdurchdringung führen. ${ }^{3}$ 
Im Duden, der den Begriff „Innovation“ in seiner Ausgabe 1915 erstmals berücksichtigt hat, findet sich eine differenziertere Definition:

1. a) (Soziologie) geplante und kontrollierte Veränderung, Neuerung in einem sozialen System durch Anwendung neuer Ideen und Techniken

b) (bildungssprachlich) Einführung von etwas Neuem; Neuerung; Reform

2. (Wirtschaft) Realisierung einer neuartigen, fortschrittlichen Lösung für ein bestimmtes Problem, besonders die Einführung eines neuen Produkts oder die Anwendung eines neuen Verfahrens

3. (Botanik) (bei ausdauernden Pflanzen) jährliche Erneuerung eines Teiles des Sprosssystems ${ }^{4}$

Für den Bibliotheks- und Informationsbereich sind die Bedeutungen von Innovation im Kontext von Soziologie, Bildungssprache und Wirtschaft zutreffend. Die Bandbreite der Innovationen spiegelt sich in den im Duden genannten Synonymen zu diesem Begriff: Erneuerung, Neuerung, Neugestaltung, Neuordnung, Neuschöpfung, Reform, Umgestaltung, Veränderung.

Bei Verwendung des Begriffs „Innovation“, insbesondere im Kontext der Wirtschaftswissenschaften, ist zu bedenken, dass bisher kein geschlossener, allgemein gültiger Innovationsansatz bzw. keine allgemein akzeptierte Begriffsdefinition vorliegt, wobei allerdings allen Definitionsversuchen folgende zwei Merkmale zugrunde liegen:

[1] Neuheit oder (Er-)Neuerung eines Objekts oder einer sozialen Handlungsweise, mindestens für das betrachtete System und

[2] Veränderung bzw. Wechsel durch die Innovation in und durch die Unternehmung, d.h. Innovation muss entdeckt / erfunden, eingeführt, genutzt, angewandt und institutionalisiert werden. ${ }^{5}$

Folgende Innovationstypen bzw. Innovationsarten sind zu unterscheiden: Produktinnovationen, Prozessinnovationen, organisatorische Innovationen, soziale Innovationen, bedarfsinduzierte Innovationen und angebotsinduzierte Innovationen; ${ }^{6}$ diese unterschiedlichen Innovationstypen bzw. Innovationsarten kommen auch im Bibliotheks- und Informationswesen zum Tragen.

Innovationen können unterschieden werden in inkrementelle bzw. in radikale Innovationen. Während die inkrementelle Innovation die Reduktion der Kosten oder eine funktionelle Verbesserung bei bestehenden Produkten, Dienstleistungen oder Prozessen anstrebt, liegt das Ziel einer radikalen Innovation in der Generierung neuer Produkte, Dienstleistungen oder Prozesse mit bislang nicht gekannten Eigenschaften. Die inkrementelle In- 
novation stützt sich auf vorhandenes Wissen und erhöht die Wettbewerbsfähigkeit in vorhandenen Märkten oder Branchen. Demgegenüber basiert die radikale Innovation auf neuem Wissen und bewirkt eine wesentliche Veränderung, die ganze Märkte oder Branchen transformiert oder auch neue kreiert. Die inkrementelle Innovation führt zu geringer Unsicherheit, während die radikale Innovation eine hohe Unsicherheit mit sich bringt. ${ }^{7}$ Für wissenschaftliche Bibliotheken, die vielfach auf eine lange Tradition zurückblicken, sind in der Regel inkrementelle Innovationen mit wenig Veränderungsrisiko attraktiver als radikale Innovationen. In jüngster Zeit gibt es allerdings durchaus Herausforderungen (Stichwort Open Access), die radikale Lösungsansätze mit sich bringen und mit denen sich auch die $\mathrm{Bi}$ bliotheken auseinandersetzen müssen.

Der Begriff „Innovation“ ermöglicht noch weitere Unterscheidungen: „Objektive Innovation“ vs. „Subjektive Innovation“ sowie „Closed Innovation“ vs. „Open Innovation“. Während der Terminus „Objektive Innovation" eine Neuerung für den relevanten Markt beschreibt, ist mit „Subjektiver Innovation" lediglich eine neue Aktivität für das Unternehmen gemeint. Bei „Closed Innovation“ agieren die Innovatoren ausschließlich innerhalb einer Organisation, während bei „Open Innovation“ externe Informationen und Kompetenzen genutzt werden. Im Weiteren werden ausschließlich Innovationen beschrieben, die in die Kategorien „Objektive Innovation“ bzw. „Open Innovation“ fallen.

Innovation meint nicht nur Entwicklung neuer Produkte, sondern insbesondere auch deren Implementierung. Mit letzterem beschäftigt sich auch die Innovationsforschung, die die Zielgruppen nach Innovationsfreude bzw. nach dem Prozess der Annahme der Innovation in fünf Gruppen unterscheidet:

- Innovatoren - die ersten 5 bis 10\%, die ein Produkt annehmen

- Early Adopters - die nächsten 10 bis $15 \%$

- Frühe Mehrheit - weitere 30\%

- Späte Mehrheit - weitere 30\%

- Laggards (Nachzügler) - verbleibende 20\%

Auch unter den wissenschaftlichen Bibliotheken in Österreich können diese Gruppen identifiziert werden, wobei bei unterschiedlichen Innovationen unterschiedliche Positionen bezogen werden können. In einem Fall kann eine Bibliothek, etwa in Form einer Produktpartnerschaft mit einem Anbieter, als Innovator agieren, in einem anderen Fall, wenn es sich um eine für die betreffende Institution weniger relevante Innovation handelt, die Innovation mit der späten Mehrheit implementieren. 
Ehe im Folgenden der Versuch unternommen wird, die aktuellen Entwicklungen sowie möglichen Perspektiven für das österreichische Bibliothekswesen zu skizzieren, lohnt sich eine Rückschau auf die Entwicklung der jüngsten Vergangenheit.

Ein wesentlicher Motor für Innovationen stellen Kooperationen von Institutionen mit gleicher oder ähnlicher Zielsetzung dar. Auch in der österreichischen Bibliothekslandschaft gibt es langjährige und tragfähige Kooperationen, die durch die Transformation von gedruckten (und elektronischen) Medien zu E-Only seit den 1990er Jahren intensiviert worden sind. In den letzten Monaten wurden einige weitere wichtige Kooperationsprojekte gestartet, die in den kommenden Jahren zu einer deutlichen Verbesserung der Bibliotheks- und Informationslandschaft führen werden.

\section{Innovationsschub durch Kooperationen bei der Transformation von gedruckten (\& elektronischen) Medien zu E-Only}

In den letzten drei Jahrzehnten setzte an den wissenschaftlichen Bibliotheken weltweit ein gravierender Veränderungsprozess ein, der charakterisiert war und ist vom Medienwandel bei den Katalogen, den Bibliografien, den Fachzeitschriften und den Fachbüchern. Die Vorteile der Transformation vom gedruckten zum elektronischen Medium sind hinlänglich bekannt und sollen hier nur kurz angerissen werden. Ein wesentliches Argument für elektronische Medien ist deren größere Verfügbarkeit, bedingt durch die Unabhängigkeit von Ort (kein Entlehnen, Verlängern bzw. Kopieren erforderlich), Zeit (Unabhängigkeit von Bibliotheksöffnungszeiten sowie Verfügbarkeit 24/7) und konkretem Objekt (kein verstelltes Buch, kein beim Buchbinder befindliches Zeitschriftenheft). Weitere ungeahnte Entwicklungsmöglichkeiten boten und bieten sich den Bibliotheken durch die mit den Online-Medien möglich gewordenen effizienteren Such- und Findemöglichkeiten - Stichworte Verlinkung, One-stop-Shop und Suchmaschinentechnologie. ${ }^{8}$ Parallel zur Entwicklung der Online-Medien wurden auch die bestehenden Kooperationen der wissenschaftlichen Bibliotheken gestärkt bzw. neue Kooperationen etabliert.

\subsection{Transformation der Kataloge}

Die Implementierung von BIBOS 2 im Jahr 1987 war die Initialzündung für die Abkehr von der bisherigen Praxis der Erfassung von Metadaten 
auf gedruckten Katalogkarten und den Beginn der gemeinsamen Onlinekatalogisierung im Österreichischen Bibliothekenverbund. ${ }^{9}$ Mit der 1999/2000 erfolgten Implementierung des Verbundbibliothekssystems Aleph 500 und der damit einhergehenden Integration der bisherigen heterogenen Teilsysteme, inbesondere BIBOS und GRIBS (bis dahin für Monografien eingesetzt) sowie der ÖZDB (bis dahin für den Zeitschriftennachweis genutzt) gab es einen weiteren Innovationsschub hin zu einem effizienteren Nachweissystem der an den Bibliotheken vorhandenen Medien. ${ }^{10}$

Mit dem Österreichischen Bibliothekenverbund wurde noch unter direkter Einflussnahme des zuständigen Bundesministeriums eine gemeinsame Infrastruktur für das wissenschaftliche Bibliothekswesen in Österreich geschaffen, die mittlerweile weit über den ursprünglichen Zweck - die gemeinsame Katalogisierung - hinaus zu einer wertvollen Plattform für tragfähige Kooperationen und wertvolle Innovationen geworden ist. ${ }^{11} 12$

\subsection{Transformation der Bibliografien}

Jahrzehntelang waren gedruckte Bibliografien eine besonders wertvolle Informationsquelle für wissenschaftliche Fachinformation. Mittlerweile sind alle großen Universalbibliografien und annähernd vollständig auch die bedeutenden Fachbibliografien, insbesondere des STM-Bereiches, digital in Form von bibliografischen Fachdatenbanken online verfügbar. Ein wichtiger Meilenstein für diese Entwicklung wurde mit der Freigabe der die medizinische Forschung dominierenden bibliografischen Fachdatenbank MEDLINE für die Öffentlichkeit im Juni 1997 gesetzt; 2004 stellte die National Library of Medicine die Herausgabe der gedruckten Version des Index Medicus überhaupt ein. ${ }^{13}$

Für die Lizenzierung von Datenbanken wurde mit der Kooperation EMedien Österreich (KEMÖ, https://www.konsortien.at/ssl/) eine geeignete Infrastruktur geschaffen, um durch den gemeinsamen Erwerb von Lizenzen in Form von Konsortien kostengünstigere Konditionen zu bekommen.

Auch im Bereich des Nachweises von Datenbanken entwickelte sich mit Etablierung und Betrieb des Datenbank-Infosystems (DBIS, http:// rzblx10.uni-regensburg.de/dbinfo/) eine Kooperation von 292 überwiegend deutschen und österreichischen Bibliotheken hinsichtlich Erfassung und Aktualisierung der Daten; DBIS umfasst 11.196 Einträge [Stand: 28.02.2015]. 


\subsection{Transformation der Zeitschriften}

Elektronische Zeitschriften kommen seit Mitte der 1990er Jahre an wissenschaftlichen Bibliotheken zum Einsatz. Nachdem bereits seit Mitte der 2000er Jahre überwiegend elektronische bzw. gedruckte \& elektronische Zeitschriften eingesetzt werden, ist derzeit ein starker Trend weg vom Doppelbezug hin zur Bezugsform E-Only zu beobachten. Als erste Universitätsbibliothek in Österreich hat die Universitätsbibliothek der Medizinischen Universität Wien mit Jahreswechsel 2012/2013 einen radikalen Umstieg bei den Fachzeitschriften vorgenommen und alle gedruckten Abonnements storniert; seither erfolgt die Literaturversorgung an der größten medizinischen Universität Österreichs ausschließlich in Form der Lizenzierung elektronischer Zeitschriften. ${ }^{14}$

Über die Kooperation E-Medien Österreich, die mit der Zielsetzung geschaffen worden ist, eine geeignete Infrastruktur für den gemeinsamen Erwerb von Lizenzen, insbesondere im Bereich der Zeitschriften, zu etablieren, werden mittlerweile wesentliche Anteile des elektronischen Zeitschriftenangebots der an der Kooperation beteiligten Bibliotheken konsortial lizenziert.

Für den Nachweis lizenzierter elektronischer Zeitschriften nutzen 624 wissenschaftliche Bibliotheken, insbesondere in Deutschland, Österreich und der Schweiz, die Elektronische Zeitschriftenbibliothek (EZB, http:// rzblx1.uni-regensburg.de/ezeit/), die einen schnellen, strukturierten und einheitlichen Zugang zu wissenschaftlichen Volltextzeitschriften im Internet bietet. Die EZB weist 79.962 Zeitschriftentitel nach [Stand: 28.02.2015].

\subsection{Transformation von Monografien}

Während sich bei Datenbanken und Fachzeitschriften in den letzten Jahren ein eindeutiger Trend - weg vom gedruckten hin zum elektronischen Medium - abgezeichnet hat, kann Vergleichbares über die Entwicklung bei Monografien, auch aus Mangel an adäquaten Angeboten seitens der Verlage, (noch) nicht festgestellt werden.

Neben elektronischen Büchern, die von einzelnen Bibliotheken direkt beim Verlag lizenziert werden, gibt es dank der Bedeutung des zugrundeliegenden Produktes Pschyrembel, einem medizinischen Nachschlagewerk, ein E-Book-Konsortium, das über die Kooperation E-Medien Österreich verhandelt und verwaltet wird. 


\section{Desiderate im österreichischen Bibliothekswesen}

Während sich die wissenschaftlichen Bibliotheken in Österreich bis zur Jahrtausendwende sehr positiv entwickelt und sich, was die Aufnahme und Implementierung von Innovationen betrifft, im internationalen Vergleich durchaus konkurrenzfähig gezeigt haben, kam es im ersten Jahrzehnt des 21. Jahrhunderts zu einer Phase, in der die österreichische Bibliothekslandschaft ins Hintertreffen zu schlittern drohte. Im Zuge der Entlassung der Universitäten in die Vollrechtsfähigkeit verzichtete das zuständige Bundesministerium auch auf eine zentrale gestalterische Rolle für die Universitätsbibliotheken und zog sich auf eine kontrollierende Rolle zurück. ${ }^{15}$ Damit einhergehend wurden auch die Möglichkeiten für eine zentrale Fördermöglichkeit für zukunftsweisende Projekte eingeschränkt, insbesondere auch eine umfassende nationale Bibliotheks- und Informationspolitik erschwert. ${ }^{16} 1718$

Die Desiderate in der österreichischen Bibliotheks- und Informationslandschaft wurden besonders deutlich, als in Deutschland mit der Schwerpunktinitiative „Digitale Information“ $2008-2012^{19}$ eine Initiative zur Weiterentwicklung der Bibliotheks- und Informationsinfrastruktur auf nationaler Ebene gestartet wurde, die mittlerweile für die Jahre 2013-201720 eine Fortsetzung gefunden hat. Ziel der Schwerpunktinitiative ist es, den weitest möglichen Zugang zu digitalen Publikationen, digitalen Daten und Quellenbeständen zu gewährleisten, optimale Voraussetzungen für die Verbreitung und Rezeption von Publikationen aus deutscher Forschung mithilfe der digitalen Medien zu schaffen, die langfristige Verfügbarkeit der weltweit erworbenen digitalen Medien und Inhalte sowie ihre Integration in die digitale Forschungsumgebung sicherzustellen sowie netzbasierte Formen des wissenschaftlichen Arbeitens durch innovative Informationstechnologien zu unterstützen. Um Wissenschafterinnen und Wissenschafter mit der bestmöglichen Informationsinfrastruktur auszustatten, die sie für ihre Forschung brauchen, wurden sechs Aktionslinien definiert:

- Nationale Lizenzierung

- Nationale Hosting-Strategie

- Forschungsprimärdaten

- Open Access

- Rechtliche Rahmenbedingungen

- Virtuelle Forschungsumgebung

Bis 2012, dem Ende der Laufzeit der 1. Phase der deutschen Schwerpunktinitiative „Digitale Information“, ist festzuhalten, dass die oben 
genannten sechs Aktionslinien in Österreich nur zum Teil und als Einzelinitiativen aufgegriffen worden sind. ${ }^{21}{ }^{22}$ Während etwa in Deutschland beträchtliche zentrale Fördermittel für Nationallizenzen zur Verfügung gestellt wurden, konnte dieses Lizenzierungsmodell in Österreich nicht realisiert werden. ${ }^{23}$

Maßnahmen zur Förderung von Open Access wurden zwar vom FWF $^{24}{ }^{25}$, der Österreichischen Akademie der Wissenschaften ${ }^{26}$ und der Universität Wien ${ }^{27}{ }^{28}$ schon relativ früh gesetzt, auf nationaler Ebene gab es allerdings lange keine gemeinsamen Initiativen, wie in einer 2012 durchgeführten Umfrage, die vom Forum Universitätsbibliotheken Österreichs an den öffentlichen Universitäten durchgeführt worden ist, deutlich geworden ist. ${ }^{29}$

\section{Aktuelle Innovationen durch Kooperationen wissenschaftlicher Biblio- theken in Österreich}

Erst in den letzten Jahren wurden wichtige Schritte gesetzt, um durch zukunftsweisende Innovationen das österreichische Bibliotheks- und Informationswesen fit für die Anforderungen der kommenden Jahre zu machen.

\subsection{Innovation Suchmaschinentechnologie}

Ziel des Einsatzes von Suchmaschinentechnologie ist die Implementierung von neuen Funktionalitäten, die eine effiziente integrierte Recherche in den Beständen der Bibliothek inklusive der lizenzierten Volltexte und Datenbanken ermöglichen.

Die Basisfinanzierung für den österreichischen Bibliothekenverbund durch das für Wissenschaft und Forschung zuständige Bundeministerium blieb seit 2000 unverändert. Deshalb konnte die angestrebte Implementierung von Suchmaschinentechnologie nur durch eigenständig zu leistende finanzielle Beiträge von interessierten Verbundbibliotheken realisiert werden. Die unbedingte Bereitschaft dieser Bibliotheken bzw. deren Trägerorganisationen machte es möglich, diese zukunftsweisende Innovation in der österreichischen Bibliothekslandschaft zu etablieren. Dem von der Österreichischen Bibliothekenverbund und Service GmbH (OBVSG, http:// www.obvsg.at/) mit der Anbieterfirma vereinbarten Rahmenvertrag sind seit 200915 Institutionen beigetreten. ${ }^{30} 31$ 


\subsection{Innovation Bibliothekssystem}

Ziel des aktuellen Verfahrens zur Ablöse des bestehenden Bibliothekssystems ist die Etablierung eines Bibliotheksverbundsystems, das aktuelle und zukünftige Anforderungen an moderne Bibliotheken besser erfüllen kann. Exemplarisch zu nennen ist die Notwendigkeit, elektronische Ressourcen besser verwalten zu können (ERM) und effiziente Nutzungsstatistiken aus dem Bibliothekssystem ohne großen Aufwand generieren zu können.

An dem seit 2014 laufenden Vergabeverfahren beteiligen sich 14 Trägerinstitutionen von wissenschaftlichen Bibliotheken; ${ }^{32}$ auch in diesem Zusammenhang ist es bemerkenswert, dass - wie schon bei der Implementierung der Suchmaschinentechnologie - keine zentrale Finanzierung möglich ist, sondern die für Anschaffung und Betrieb des neuen Systems benötigten Mittel von den beteiligten Institutionen bereitgestellt werden.

\subsection{Innovationen Kooperation E-Medien Österreich}

Ziel der Kooperation E-Medien Österreich (KEMÖ, https://www.konsortien.at/) ist die möglichst kosteneffektive konsortiale Lizenzierung von elektronischen Informationen. Die 2005 gegründete Kooperation wird jeweils für eine Laufzeit von drei Jahren abgeschlossen und hat in der Vergangenheit die Bereitstellung von Fachinformation in Form von Datenbanken und elektronischen Zeitschriften für Wissenschaft und Forschung in Österreich maßgeblich gestaltet. ${ }^{33} 3435$ Aktuell sind 55 Institutionen Partner der Kooperation. Mit Jänner 2015 ist ein neuer Dreijahresvertrag für die Kooperationsperiode 2015-2017 in Kraft getreten.

Die Besonderheit der Kooperation, die räumlich und organisatorisch an der OBVSG angesiedelt ist, liegt darin, dass die Kooperationspartner die Einrichtung und den Betrieb einer Geschäftsstelle der Kooperation mit ihren Beiträgen ermöglichen. Für die nächste Kooperationsperiode wurde vereinbart, im Hinblick auf zusätzliche Aufgaben der Geschäftsstelle deren personelle Ausstattung um eine weitere Stelle auf insgesamt 3 FTEs zu erweitern.

\subsection{Innovation E-Infrastructures Austria}

2012 wurde vom Bundesministerium für Wissenschaft und Forschung beschlossen, Hochschulraum-Strukturmittel im Umfang von 63 Mio. Euro zur Anschubfinanzierung von universitären Kooperationsvorhaben zur Verfü- 
gung zu stellen (BGBI. II Nr. 292/2012); aus diesen Mitteln können Projekte bis zu einem Drittel finanziell gefördert werden. Insgesamt wurden 63 Mio. Euro für Kooperationsprojekte in der Lehre Forschung / Entwicklung und Erschließung der Künste (48 Mio. Euro) sowie in der Verwaltung (15 Mio. Euro) zur Verfügung gestellt. ${ }^{36}$

Von 33 Projektanträgen im Bereich der Verwaltung wurden 22 Projekte, davon zwei Bibliotheksprojekte - neben „E-Infrastructures Austria“ noch das Projekt „Entwicklung und Umsetzung eines Beschaffungsprozesses für österreichische Universitätsbibliotheken" - bewilligt.

Das Hochschulraumstrukturmittelprojekt „E-Infrastructures Austria“ (http://www.e-infrastructures.at/) bietet seit seinem Start im Jänner 2014 ausgezeichnete Rahmenbedingungen, um für zwei wichtige Themen, die auch in der deutschen Schwerpunktinitiative „Digitale Information“ angeführt werden (Open Access und Forschungsdaten), zukunftsweisende Lösungen auf nationaler Ebene zu entwickeln. In diesem Zusammenhang ist eine frühere, letztlich erfolglose Initiative zur gesetzlichen Verankerung von Open Access in der Novelle zum Universitätsgesetz 2009 anzuführen. Darin war beschlossen worden, eine „Zentrale Datenbank für wissenschaftliche und künstlerische Arbeiten" aufzubauen, die als digitales Repositorium an der Österreichischen Bibliothekenverbund und Service $\mathrm{GmbH}$ betrieben werden sollte. Im Budgetbegleitgesetz 2010 wurde die ein Jahr zuvor beschlossene zentrale Datenbank als eine von vielen aufgrund der Wirtschaftskrise 2008 notwendig gewordenen Einsparungsmaßnehmen wieder abgeschafft. ${ }^{37}$

Mit der Förderung des Hochschulraumstrukturmittelprojektes „E-Infrastructures Austria" durch das zuständige Bundesministerium ist nun eine fundierte Auseinandersetzung mit den zukunftsweisenden Themen Open Access und Forschungsdaten auf nationaler Ebene gesichert ${ }^{38}$; das Projekt hat eine Laufzeit von drei Jahren (2014-2016) und gliedert sich in drei Teilprojekte, die in zwölf Work Packages bearbeitet werden. Ziele von „E-Infrastructures Austria“ sind der Aufbau von lokalen Dokumentenservern, die Konzeption und Aufbau von Repositorien-Infrastrukturen für Forschungsdaten und andere komplexe Datenbestände und der Aufbau des Wissensnetzwerks „E-Infrastructures Austria“.39 40

\subsection{Innovation Open Access Network Austria}

Im November 2012 wurde unter der Schirmherrschaft der Österreichischen Universitätenkonferenz (UNIKO) und des FWF Der Wissenschaftsfonds, die als erste österreichische Institutionen die „Berliner Erklärung über of- 
fenen Zugang zu wissenschaftlichem Wissen" unterzeichnet haben, das Open Access Network Austria (OANA, http://www.oana.at) gegründet ${ }^{41}$, das folgende Ziele verfolgt:

- Abstimmung der und Empfehlungen zu OA-Aktivitäten der österreichischen Forschungsstätten, Fördergeber und Forschungspolitik (inkl. der Berücksichtigung internationaler Entwicklungen)

- Positionierung gegenüber den Informationsanbietern (v.a. Verlage)

- Ansprechpartner und Informationsquelle für Wissenschaftlerinnen und Wissenschaftler, Forschungsstätten und (Forschungs-) Politik

Um Open Access in Österreich nachhaltig zu etablieren wurden 2014 fünf Arbeitsgruppen zur Erstellung von Empfehlungen eingerichtet, die OANAMitglieder dabei unterstützen sollen, eine effiziente Open Access-Strategie zu entwickeln (Open Access Policy, Finanzierung von Open Access, Rechtliche und politische Rahmenbedingungen, Publikationsmodelle, Einbindung von Wissenschafterlnnen).

\section{Innovationen als Zukunftschance für Bibliotheks- und Informations- infrastruktur in Österreich}

Im Rückblick kann die kooperative Einführung und Weiterentwicklung von Innovationen an den wissenschaftlichen Bibliotheken in Österreich in den zurückliegenden Jahrzehnten durchaus als Erfolg bezeichnet werden. Nachdem etliche Jahre aber eher von Pessimismus getragen waren, was die Möglichkeit der Implementierung innovativer Services und Ressourcen betrifft, so wurden in den letzten zwei Jahren wichtige Innovationen angestoßen. ${ }^{42}$

Die kommenden Herausforderungen in einem Umfeld globalen Wettbewerbs werden eine weitere Intensivierung der Kooperationen von Bibliotheks- und Informationseinrichtungen zwingend erforderlich machen. Wesentlich für die Zukunftsfähigkeit der Bibliotheks- und Informationsinfrastruktur in Österreich wird es auch sein, relevante Themen rascher aufzugreifen und zu bearbeiten. Gelangte der Autor des vorliegenden Beitrages bei seinem im Rahmen der ODOK 2010 in Leoben gehaltenen Abschlussvortrag $^{43}$ aufgrund der Desiderate im Hinblick auf Innovationsmöglichkeiten für die österreichische Bibliotheks- und Informationsinfrastruktur zu einer eher pessimistischen Einschätzung, so konnte er nach Analyse der aktuellen Entwicklung seit 2010 in seinem im Rahmen der ODOK 2014 in Zell am See gehaltenen Eröffnungsvortrag ${ }^{44}$ eine nunmehr optimistischere 
Perspektive für Gegenwart und unmittelbare Zukunft präsentieren. Es bleibt zu hoffen, dass bei der ODOK 2018 darüber zu berichten sein wird, wie die an den aktuellen Innovationsprojekten beteiligten Bibliotheken und Institutionen die aktuellen Chancen für eine Optimierung der Bibliotheks- und Informationsinfrastruktur in Österreich erfolgreich genutzt haben werden.

Mag. Bruno Bauer Universitätsbibliothek der Medizinischen Universität Wien Währinger Gürtel 18-20, A-1097 Wien E-Mail: bruno.bauer@meduniwien.ac.at Website: http://ub.meduniwien.ac.at

1 ODOK 2014 - Österreichisches Online-Informationstreffen und Österreichischer Dokumentartag: Call for Papers: ODOK 2014 - Innovationen in der Medienwelt, 17.-19. September 2014, Zell am See. Online im Internet: http://www.odok.at/dokumente/2014/odok/ODOK 2014 CallforPapers.pdf [30.11.2014]

2 Konrad Umlauf, Stefan Gradmann, Peter Lohnert (Hrsg.): Lexikon der Bibliotheks- und Informationswissenschaft. Band 1: A bis J. Stuttgart: Hiersemann 2011.

3 Innovation. In: Wikipedia: Die freie Enzyklopädie. Online im Internet: http://de.wikipedia.org/wiki/Innovation [30.11.2014]

4 Innovation. In: Duden online. Online im Internet: http://www.duden. de/rechtschreibung/Innovation [30.11.2014]

5 Martin G. Möhrle, Dieter Specht: Innovation. In: Gabler Wirtschaftslexikon. Online im Internet: http://wirtschaftslexikon.gabler.de/Definition/innovation.html [30.11.2014]

6 Innovation. In: Wirtschaftslexikon. Online im Internet: http://www. wirtschaftslexikon24.com/d/innovation/innovation.htm [30.11.2014]

7 ZIT - Die Technologieagentur der Stadt Wien GmbH (Hrsg.): Bewertungssystem Innovation. Stand April 2010. Online im Internet: http:// www.zit.co.at/fileadmin/user upload/ZIT/Foerderungen/Innovation/ Bewertungssystem Innovation 122010.pdf [30.11.2014]

8 Eveline Pipp: Das E-Medien-Angebot österreichischer Universitätsbibliotheken. In: Bruno Bauer, Christian Gumpenberger, Robert Schiller (Hrsg.): Universitätsbibliotheken im Fokus: Aufgaben und Perspektiven der Universitätsbibliotheken an öffentlichen Universitäten in Österreich: Graz - Feldkirch: Neugebauer, 2013. S. 142-153. 
9 Eva Bertha: Bibliotheksautomation an österreichischen Universitätsbibliotheken. In: Bruno Bauer, Christian Gumpenberger, Roberts Schiller (Hrsg.): Universitätsbibliotheken im Fokus: Aufgaben und Perspektiven der Universitätsbibliotheken an öffentlichen Universitäten in Österreich: Graz -Feldkirch: Neugebauer, 2013. S. 130-141.

10 Wolfgang Hamedinger: Der große Wechsel: von Bibos zu Aleph 500. In: Klaus Niedermair (Hrsg.): Mitteilungen der Vereinigung Österreichischer Bibliothekarinnen \& Bibliothekare 52 (1999), H. 1, S. 38-42.

11 Wolfgang Hamedinger: 10 Jahre Verbundorganisation neu: Verbundfamilie, Verbundarbeit, Kooperation. In: Klaus Niedermair (Hrsg.): Die neue Bibliothek. Anspruch und Wirklichkeit. 31. Österreichischer Bibliothekartag, Innsbruck 2011. Graz-Feldkirch: Neugebauer, 2012. S. 173-178.

12 Peter Seitz: Die Universitätsbibliotheken aus der Perspektive des Bundesministeriums für Wissenschaft und Forschung. In: Bruno Bauer, Christian Gumpenberger, Roberts Schiller (Hrsg.): Universitätsbibliotheken im Fokus: Aufgaben und Perspektiven der Universitätsbibliotheken an öffentlichen Universitäten in Österreich: Graz -Feldkirch: Neugebauer, 2013. S. 239-247.

13 Bruno Bauer: Medizinbibliotheken als Treibervon Innovationen für die Digitale Bibliothek: Metamorphose von wissenschaftlichen Bibliotheken am Beispiel der Universitätsbibliothek der Medizinischen Universität Wien. In: GMS Medizin Bibliothek Information 13 (2013), H. 1-2, Doc14.

14 Bruno Bauer, Helmut Dollfuß, Daniel Formanek: Universitätsbibliothek der Medizinischen Universität Wien goes e-only: Umstellung des Zeitschriftenbezugs von p+e auf e-only ab 1. Januar 2013. In: GMS Medizin Bibliothek Information 13 (2013), H. 3, Doc20.

15 Bruno Bauer: Nationale und internationale Kooperationen der österreichischen Universitätsbibliotheken 2007. In: Mitteilungen der Vereinigung Österreichischer Bibliothekarinnen \& Bibliothekare 61 (2008), H. 2, S. 21-31.

16 Bruno Bauer: Braucht Österreich eine Schwerpunktinitiative „Digitale Information"? Status quo und Perspektiven für die Hochschulbibliotheken. In: Mitteilungen der Vereinigung Österreichischer Bibliothekarinnen \& Bibliothekare 65 (2012), H. 3/4, S. 394-415.

17 Bruno Bauer, Peter Klien: „Wenn man die Geschichte aller Wissenschaften und des Wissens überhaupt betrachtet, war die Bibliothek immer ein zentraler Ort" - Interview mit Bundesminister o.Univ.-Prof. Dr. Karlheinz Töchterle. In: Mitteilungen der Vereinigung Österreichischer Bibliothekarinnen \& Bibliothekare 65 (2012), H. 3/4, S. 341351. 
18 Bruno Bauer, Edith Stumpf-Fischer: Die politische Rolle der Wissensspeicher und -vermittler Bibliotheken. Potenzial und Perspektiven für klassische Think Tanks. - In: Köhler, Thomas ; Mertens, Christian (Hrsg.). Jahrbuch für politische Beratung 2010/2011: Eine klassische Alternative. Wien - Köln - Weimar: Böhlau, 2011.S. 165-180.

19 Schwerpunktinitiative „Digitale Information“ der Allianz-Partnerorganisationen. Berlin, 11. Juni 2008. Online im Internet: http://www. allianzinitiative.de/fileadmin/user upload/redakteur/pm allianz digitale information details 080612.pdf [30.11.2014]

20 Schwerpunktinitiative „Digitale Information“ der Allianz der deutschen Wissenschaftsorganisationen Fortsetzung der Zusammenarbeit in den Jahren 2013 bis 2017. München, den 26. Juni 2012. Online im Internet: http://www.allianzinitiative.de/fileadmin/user upload/redakteur/ Schwerpunktinitiative 2013-2017.pdf [30.11.2014]

21 Bruno Bauer, Kerstin Stieg: Open Access Publishing in Österreich 2010. In: Bibliotheksdienst 44 (2010), H. 7, S. 700-710.

22 Bruno Bauer, Michael Katzmayr, Karlo Pavlovic, Kerstin Stieg: Open Access in Österreich - Anmerkungen zur aktuellen Entwicklung und zu den Perspektiven für einen Paradigmenwechsel im wissenschaftlichen Publikationswesen. In: Mitteilungen der Vereinigung Österreichischer Bibliothekarinnen \& Bibliothekare 65 (2012), H. 2, S. 155-166.

23 Bruno Bauer: Nationallizenzen - ein Desiderat in Österreich. In: GMS Medizin Bibliothek Information 7 (2007), H. 2, Doc33.

24 Falk Reckling: Eine freie Wissenschaft braucht die freie Zirkulation ihrer Erkenntnisse. Zur aktuellen Entwicklung von Open Access aus der Perspektive des Fonds zur Förderung der wissenschaftlichen Forschung (FWF). In: Klaus Niedermair (Hrsg.): Die neue Bibliothek. Anspruch und Wirklichkeit. 31. Österreichischer Bibliothekartag, Innsbruck 2011. Graz - Feldkirch: Neugebauer, 2012. S. 102-112

25 Falk Reckling: Open Access - Aktuelle internationale und nationale Entwicklungen. 20.02.2013. Online im Internet: http://www.fwf.ac.at/ fileadmin/files/Dokumente/News Presse/News/FWF OA-2013.pdf [30.11.2014]

26 Michael Nentwich, Herwig Stöger, Vittorio Muth: Open Access und die Österreichische Akademie der Wissenschaften. Mitteilungen der Vereinigung Österreichischer Bibliothekarinnen \& Bibliothekare 65 (2012). H. 2, S. 178-186.

27 Susanne Blumesberger, Gerda McNeill: Mitteilungen der Vereinigung Österreichischer Bibliothekarinnen \& Bibliothekare 65 (2012), H. 2, S. 187-199. 
28 Nora Schmidt, Wolfram Seidler: Das institutionelle Repositorium der Universität Wien. Mitteilungen der Vereinigung Österreichischer Bibliothekarinnen \& Bibliothekare 65 (2012), H. 2, S. 218-221.

29 Bruno Bauer, Christian Gumpenberger, Ingrid Haas, Michael Katzmayr, Eva Ramminger, Doris Reinitzer: Open Access Bestandsaufnahme an österreichischen Universitäten: Ergebnisse einer Umfrage im Auftrag des Forums Universitätsbibliotheken Österreichs (UBIFO). In: Mitteilungen der Vereinigung Österreichischer Bibliothekarinnen \& Bibliothekare 66 (2013), H. 3/4, S. 535-558.

30 Markus Knitel: Die konsortiale Primo-Instanz der OBVSG im Überblick. In: Mitteilungen der Vereinigung Österreichischer Bibliothekarinnen \& Bibliothekare 64 (2011), H. 2, S. 192-195.

31 Wolfgang Hamedinger: Aktuelle und künftige Verbundentwicklungen: Einheit in der Vielfalt. In: Klaus Niedermair (Hrsg.): Die neue Bibliothek. Anspruch und Wirklichkeit. 31. Österreichischer Bibliothekartag, Innsbruck 2011. Graz -Feldkirch: Neugebauer, 2012. S. 179-196.

32 Wolfgang Hamedinger: Auf die Plätze, fertig, los. Bekanntmachung zur Ausschreibung „Aleph-Nachfolge“ veröffentlicht. In: obv sg newsletter 2/2013, S. 2. Online im Internet: http://www.obvsg.at/uploads/media/newsletter-obvsg-2013-2.pdf [30.11.2014]

33 Kerstin Stieg, Karlo Pavlovic: Kooperative Lizenzierung von OnlineRessourcen in Österreich. In: Mitteilungen der Vereinigung Österreichischer Bibliothekarinnen \& Bibliothekare 63 (2010), H. 3/4, S. 90-94.

34 Kerstin Stieg: Kooperation E-Medien Österreich als Partner der Universitätsbibliotheken. In: Bruno Bauer, Christian Gumpenberger, Roberts Schiller (Hrsg.): Universitätsbibliotheken im Fokus: Aufgaben und Perspektiven der Universitätsbibliotheken an öffentlichen Universitäten in Österreich: Graz -Feldkirch: Neugebauer, 2013. S. 257-266.

35 Bruno Bauer, Helmut Hartmann: Bibliothekskonsortien in Österreich: Helmut Hartmann, Pionier für Konsortien elektronischer Medien in Österreich und erster Leiter der Kooperation E-Medien Österreich, beantwortet 11 Fragen von Bruno Bauer. In: Mitteilungen der Vereinigung Österreichischer Bibliothekarinnen \& Bibliothekare 66 (2013), H. 3/4, S. 422-447.

36 Bundesministerium für Wissenschaft und Forschung: Kooperationsausschreibung 2013 / Auswahlkriterien. Online im Internet: https:// hrsm-koop2013.bmwf.gv.at/index.php?option=com content\&view=a rticle\&id=4\&Itemid=16 [30.11.2014]

37 Josef Pauser: Error! - „Zentrale Datenbank für wissenschaftliche und künstlerische Arbeiten" wurde Ende 2010 wieder beerdigt. In: Mittei- 
lungen der Vereinigung Österreichischer Bibliothekarinnen \& Bibliothekare 64 (2011), H. 1, S. 120-124.

38 Bruno Bauer, Michael Birkner, Andrea Braidt, Andreas Ferus, Michaela Glanz, Eva Ramminger, Lisa Schilhan, Werner Schlacher, Maria Seissl und Ute Weiner: e-Infrastructures Austria - aus der Perspektive beteiligter Organisationen. In. Mitteilungen der Vereinigung Österreichischer Bibliothekarinnen \& Bibliothekare 67 (2014), H. 2, S. 205-214.

39 Barbara Sánchez Solís: e-Infrastructures Austria. Mitteilungen der Vereinigung Österreichischer Bibliothekarinnen \& Bibliothekare 67 (2014), H. 2, S. 195-204.

40 Bruno Bauer, Paolo Budroni, Andreas Ferus, Raman Ganguly, Eva Ramminger und Barbara Sanchez Solis: e-Infrastructures Austria 2014: Bericht über das erste Jahr des Hochschulraumstrukturmittelprojekts für den koordinierten Aufbau und die kooperative Weiterentwickung von Repositorieninfrastrukturen. In: Mltteilungen der Vereinigung Österreichischer Bibliothekarinnen \& Bibliothekare 68 (2015), H. 2, S. $X X X X X X X X X X X X X X X$

41 Bruno Bauer: Konstituierung von OANA (Open Access Netzwerk Austria) am 21.11.2012. In: Mitteilungen der Vereinigung Österreichischer Bibliothekarinnen \& Bibliothekare 66 (2012), H. 2, S. 362-365.

42 Bruno Bauer: Open-Access-Kooperationen in Österreich: Open Access Network Austria und E-Infrastructures Austria - aktuelle Entwicklungen seit 2012. In: GMS Medizin Bibliothek Information 14 (2014), H. 3, Doc22.

43 Bruno Bauer: Braucht Österreich eine Schwerpunktinitiative »Digitale Information«? In: ODOK 2010 [Abstracts]. Online im Internet: http:// www.odok.at/dokumente/2010/odok/ODOK 2010 Abstracts.pdf

44 Bruno Bauer: Innovationen an österreichischen Bibliotheken - Status und Perspektive aktueller Kooperationsprojekte [Abstracts]. Online im Internet: http://www.odok.at/dokumente/2014/odok/ODOK_2014_ Abstracts.pdf

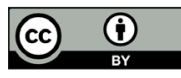

Dieses Werk ist lizenziert unter einer Creative-Commons-Lizenz Namensnennung 3.0 Österreich. 